\title{
Towards a Solution to Create, Test and Publish Mixed Reality Experiences for Occupational Safety and Health Learning: Training-MR
}

\author{
Miguel A. Lopez ${ }^{1}$ Sara Terron¹, J. M. Lombardoํ, Rubén Gonzalez-Crespo ${ }^{2}$ * \\ ${ }^{1}$ Fundación I+D del software libre (FIDESOL), Granada (Spain) \\ ${ }^{2}$ Universidad Internacional de La Rioja, Logroño (Spain)
}

Received 29 April 2021 | Accepted 1 July 2021 | Published 23 July 2021

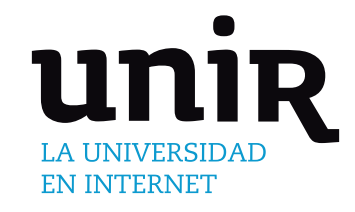

\section{ABSTRACT}

KEYWORDS

Artificial intelligence, Internet of Things, Human Augmentation, virtual reality, or mixed reality have been rapidly implemented in Industry 4.0, as they improve the productivity of workers. This productivity improvement can come largely from modernizing tools, improving training, and implementing safer working methods. Human Augmentation is helping to place workers in unique environments through virtual reality or mixed reality, by applying them to training actions in a totally innovative way. Science still has to overcome several technological challenges to achieve widespread application of these tools. One of them is the democratisation of these experiences, for which is essential to make them more accessible, reducing the cost of creation that is the main barrier to entry. The cost of these mixed reality experiences lies in the effort required to design and build these mixed reality training experiences. Nevertheless, the tool presented in this paper is a solution to these current limitations. A solution for designing, building and publishing experiences is presented in this paper. With the solution, content creators will be able to create their own training experiences in a semiassisted way and eventually publish them in the Cloud. Students will be able to access this training offered as a service, using Microsoft HoloLens2. In this paper, the reader will find technical details of the Training-MR, its architecture, mode of operation and communication.

\author{
Human Augmentation, \\ Mixed Reality, Virtual \\ Reality, Education, \\ Elearning.
}

\section{INTRODUCTION}

$\mathrm{N}$ EW technologies are transforming the society in which we live at a breakneck pace. The application of new technologies takes place in various areas in order to improve productive processes, facilitate personal relationships, or help to better understand society. This global transformation reaches its full potential in transforming the more traditional industry into the new 4.0 industry which is already a reality [1] [2]. Sensors networks, embedded systems, or wearable devices networks are interconnected to form large IoT networks (Internet of Things). These IoT networks have enhanced the interoperability of companies [3]. In addition, new artificial intelligence technologies have been incorporated to a large extent in the industry, empowering the use of data to optimize, automate and improve various types of processes [4]. This ecosystem is in continuous technological revolution, which encourages other scientific fields to be growing strongly. Technologies related to "Human Augmentation" (HA) seek to offer technological solutions to improve people's productivity by

\section{* Corresponding author.}

E-mail addresses: malopez@fidesol.org (M. A. López), sterron@ fidesol.org (S. Terron), jmlombardo@fidesol.org (J. M. Lombardo), ruben.gonzalez@unir.net (R. Gónzalez-Crespo). using different tools and algorithms. A subset of these tools are those encompassed by the "continuum reality" [5]. This concept proposes other realities that are accessible through the use of new technologies. In this way, users can experience situations and perform actions different from the real ones, depending on the position in which we are in the "continuum reality". Within this area of research, virtual reality is experiencing an exponential growth in recent years [6]. The possibility of placing the user in a controlled, completely real and highly interacting environment has encouraged many researchers to explore the applicability in engineering [7], medicine [8], or education [9], among others. In this case, users interact with virtual elements. Virtual reality is not the only alternative explored by researchers. The rise of smartphones with high computing power and a camera with appropriate technical features, provides a perfect platform for the execution of many augmented reality solutions [10]. Digital elements are represented in the user's visible spectrum through an external element. These elements can interact with each other or as a result of user actions. The development of the latest hardware platforms by the large manufacturers of the technology industry has been made possible by mixed reality to assist in software development, which have been applied in industry, architecture, engineering or construction [11] [12]. For all the so-called extended realities, researchers have studied how these technologies could be applied to training and education, in order to enhance students' performance and skills. With regard to training, particularly for industry, the focus on occupational risks 
prevention should be highlighted. Occupational Safety and Health (OSH) is a major challenge for society and science. In 2016, about 3 million workers in the industry sector reported an occupational injury or illness, which is equivalent to $2.9 \%$ of full-time workers registered in the United States [13]. The science still has a long way to go to clearly and correctly identify the factors that can cause occupational accidents or professional illnesses. Within the scope of interest of this paper, we find research related to the use of extended realities applied in the training of workers, such as virtual reality or mixed reality. Specifically, we propose our solution, whose objective is the democratization of training with extended realities through technologies applied to the prevention of occupational risks. This solution offers a cloud service for terminals of different technologies with which students may experience situations of risk, but without compromising their physical integrity or implying any cost in materials, and without causing possible damage to the company's facilities or resources. This type of student-centered training will help to improve their rapid response to emergency situations, as well as to know the protocols to be followed in order to carry out the work in appropriate conditions of safety and health. The training activity can be performed in a delocalized way with virtual reality or on site with mixed reality. Furthermore, to break the barrier of the cost of applying this technology [14] [15] [16], our development offers a set of tools for the creation of training experiences. A trainer can use these tools to build their own mixed reality experiences in a completely customized way depending on the workplace.

This article is structured as follows: the background when the authors introduce the library review. Then, in motivation and methodology we present the result of the OSH analyst and the Training-MR objective. We continue with the technical description where we resume the main issues, details, characteristics about Training-MR. At the end, discussion and conclusion are presented where we analyse the advantages of the Training-ME and present its limitations and future lines.

\section{BACKGROUND}

Human augmentation comprises a field of science whose objective is to improve human capacities through the use of tools, which can have a different degree of integration with people's actions and perceptions of their environment. A common example of these tools is the devices used by people with reduced hearing capacity. Human augmentation can be differentiated from other similar fields of research such as Human Enhancement (HE), in which the improvement of the human body itself is pursued. That is, HE seeks to improve the human body through the use of various technologies [17], while HA focuses on the application of technologies to improve human capacity and productivity, without the need to modify the body itself. An example of this differentiation is found in the current use of mixed reality glasses that provide real-world digital information, in front of a hypothetical artificial eye that sends digital and real information to the user's brain. In this case, the use of mixed reality glasses corresponds to HA and the artificial eye with HE. Providing a clear definition for HA is not easy, so several definitions of this concept can be found in the scientific literature [18]. Li introduces human augmentation technology referred to "methods with which human beings can obtain abilities exceeding the normal level or can compensate for abilities impairments" [19]. Another main definition is that provided by Rasiano [20]. In their study it is presented as "an interdisciplinary field that addresses methods, technologies and their applications for enhancing sensing, action and/or cognitive abilities of a human. This is achieved through sensing and actuation technologies, fusion and fission of information, and Artificial Intelligence methods".
The study of HA is often divided to improve its understanding and study. Li proposes a classification according to the scientific field and the impact of the adopted technology on the user. The four categories identified are as follows [19]:

- Medication augmented: for research focusing on the use of medication.

- Genetic augmented: for research using genetic modification techniques.

- Mechanical augmented: for research that proposes the use of hardware or electronics.

- Surgical augmented: for research focused on surgical operations of patients.

Other categories of HA have been proposed, such as Rasiano's research explaining three categories according to augmented skill [20]:

- Sense augmented: improvement of the user's ability to perceive the world.

- Action augmented: improvement of the user's performance capabilities.

- Cognition augmented: improvement of the cognitive abilities of the user.

Based on the numerous considerations that can be found in the literature and the studies carried out in this field, we define Human Augmentation as "the augmentation of the user from devices with which they are equipped or dressed, to improve the results of tasks by transforming the way they are performed". Thus, a categorization of the human augmentation is proposed with an approach that is not based on the augmented user capacity or the way in which this is achieved. Today, and with a foreseeable increase in the future, this augmentation will be achieved by several means (Li's proposal [19]), and will affect several capacities (Raisamo's proposal [20]). Accordingly, it is already common to find a single device that influences both senses and actions (the Microsoft HoloLens, for example). Taking this into account, we offer our own categorization based on the augmentation achieved by the user from the technology implemented for this purpose:

- Augmented successfully: the application of one or more HA technologies enable a user to perform tasks that would otherwise be unfeasible, or even achieve a more efficient performance. An example is the combination of mixed reality glasses with hand detection devices and a remote robot to perform underwater operations.

- Augmented Multitasking: the HA allows a user to perform parallel operations, which would otherwise have to be done sequentially. This would be the case, for example, of executing a complex task in an industrial production chain with two collaborative robots, one operated by voice commands and the other by hand gestures.

- Augmented perception: set of HA devices that provide information to the user about the environment around them. This category groups all technologies focused on new design, creation or research modes, in which information and data are the main object of actions. For example, a scientific experiment conducted with mixed reality simulations.

Focusing on the application of these technologies in the field of occupational safety and health, some studies can be found.In 2012, the EUSafe project was created in Europe to promote the study on the prevention of occupational risks, supporting research in this área [21]. In this regard, education and training of all the roles involved in the occupational risk prevention chain plays a key role, including from auditors and inspectors in preventive matters to the workers themselves [21] [22]. In response to the needs of society and international research, a number of new technologies have 


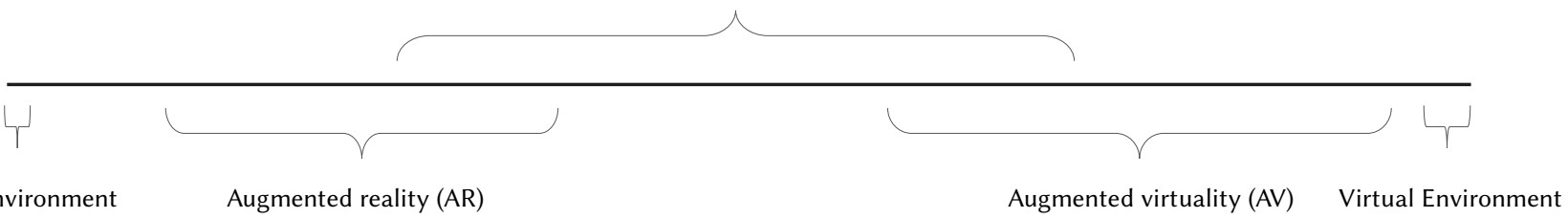

Real Environment

Augmented reality (AR)

Augmented virtuality (AV)

Virtual Environment

Fig. 1. Virtual continuum schema from Milgran y Kishino [5].

been successfully applied. Using artificial intelligence, we find the study of Simeone et al. in which they address the construction of a cloud platform for monitoring workers, in order to avoid accidents or injuries resulting from their usual work [23]. The application of Internet of Things (IoT) technologies and principles for the protection of workers also corresponds to another well-explored scientific area. Sensorizing workers' behavior has benefited workplace safety, as research by Suganya et al. shows, which exposes the construction of a miner monitoring system that includes various personal sensors and equipment to ensure their safety [24]. Another IoT study for the protection of workers in coal mines is conducted by Kumar et al. [25]. One of the main applications of IoT technologies in this area focuses on the helmet of workers. This individual protection element has unique characteristics (type-approval, obligation to use, position and guidance vis-à-vis the user). Thus, these protection elements become an integration hub for IoT sensors and devices deployed on the workers themselves [26] [27].

Considering the technologies that the HA encompasses, this paper focuses on the extent of reality, so we aim to improve the user's ability to perceive and interact with the surrounding environment. There are several types of applications of these realities, which depend on the digital tools selected. In this regard, Milgran and Kishino proposed the so-called "virtual continuum" [5]. This "virtual continuum" corresponds to the linear representation presented in Fig. 1, in which the technology is located according to its proximity to the real world (free of digital elements), or virtual (where every user-perceptible element is digital). There is a very wide range of possibilities between the two ends of the line. Augmented reality is the exposure of user-perceptible digital elements within their environment. An example of everyday use can be a vehicle browser application that expands the user's perception of his environment, providing the user with the appropriate direction. On the other hand, the increase in the load of digital elements while reducing the user's perception of their real environment, lead to the approach towards extended virtual reality. Mixed reality, meanwhile, occupies a distinct position as it displays digital 3D elements without removing user perception from the real environment. For the purpose of this study, we consider two positions within the "virtual continuum" to be of interest. The first of these is the virtual reality, positioned on the far right of the image, corresponding to the virtual environment, in which only digital elements are perceived by the user. The user feels immersed in a virtual environment. The second is the mixed reality, in which users visualize and interact with digital elements while still perceiving the real world. In mixed reality, digital objects can interact with each other or with other objects in the real world.

\section{A. Virtual Reality}

Virtual reality (VR) was introduced in the 1960s, experiencing various modifications over the years due to the advancement of science and technology. Gigante in 1993 identified virtual reality as "the illusion of participation in a synthetic environment rather than external observation of such an environment. VR relies on threedimensional (3D), stereoscopic, head-tracked displays, hand/body tracking and binaural sound. VR is an immersive, multisensory

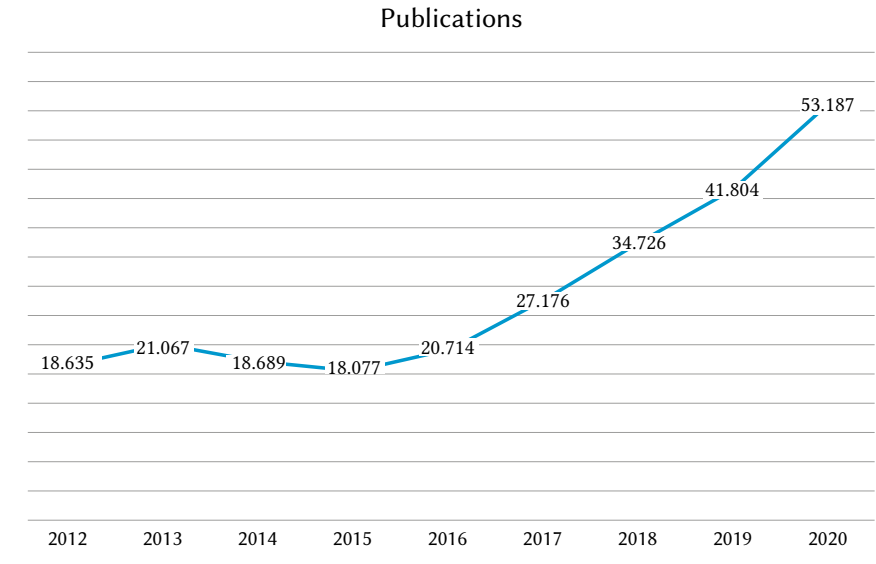

Fig. 2. Chart of papers with virtual reality key word. Data from https://app. dimensions.ai/.

experience" [28]. On the other hand, in 2019 Kardong-Edgren states the need to agree on a term that today meets the variety of applications and research carried out around this concept [29]. The research in HA and VR is currently at a time of great relevance for their research. Technological progress has led to the lower price of technology, which has turned VR glasses into a common consumer product for the general population. This has influenced the conduct of applicability studies in different fields of research and the consequent increase in the number of scientific publications. As Fig. 2 shows, the number of scientific papers containing the keyword "virtual reality" continues to grow over the years. The increase in published scientific work reflects the opportunities offered by these technologies. Isolating a user in a simulated, virtual, and 3D environment from the real world is a useful tool. Also, it is worth noting the degrees of freedom available to a user in virtual reality. The most commonly used devices such as Oculus Quest or HTC VIVE are offered as elements of interaction with the virtual environment. The set of "head mounted displays" (HMD) and hand tracking devices in VR offer the user freedom related to: (1) head tracking, i.e. the user's view in the real environment is driven by the movement of their head; (2) wrist rotation, so that virtual hands rotate with the movement of the user's wrist; (3) head and hand orientation are interpolated to estimate a natural position of the arms, although in most virtual environments the user's arms are removed from simulation; (4) the movement of three of the five fingers of each hand are detected by virtual reality hand tracking devices and sent to the digital fingers, interpolating the remaining two fingers to provide a feeling close to reality. It is common for current HMD devices to have a way to recognize the depth of field in a particular configured environment, which in Oculus Quest is called The Guardian. This provides the user with a controlled environment where their position is detected at all times, giving freedom of movement, also taking into account their height to know if they are crouched or standing. Thus, with modern systems, a user in a virtual world can simulate a controlled movement in a given space and even interact through the height of his gaze, detected by the movement and position of the head. As far as the 
interaction with the hands is concerned, the sensation experienced is quite similar to the reality, since the most common movements that a person makes, such as picking, pressing, releasing or pinching with his thumb and forefinger, are perfectly detected. These virtual immersion features have popularized its use. The application of virtual reality in medical research has been very relevant, given the trajectory of this technology for simulation. Quigley et al. conducted a study on the use of VR for the training of patients aimed at weight loss [30]. Lombardo and López, both authors of this paper, also explored the application of VR in support of the rehabilitation of Parkinson's patients [8]. Similarly, many studies highlight the pedagogical virtues of the use of these tools [31] [32] [33] [34]. Different levels of application of virtual reality in the education and training of people can be found. A first level is identified with the implementation in basic education [35]. In more advanced studies such as those at the university, we find the research of Porter et al., in which they concluded that the use of VR improved the performance of the students, compared to those who were trained using only books or videos [36]. Du et al. performed a study with two different types of training experiences in VR, in which one group of students participated in the virtual experience individually, while in the other group more than one student was connected in the same experience and could interact with each other [9]. The results showed that students who participated in both types of training experiences with VR obtained better qualifications than those who used traditional methods. It is not difficult to find in the scientific literature studies that apply virtual reality in training oriented to the industrial sector, given the virtues of its adoption. VR has been used in different sectors to train professionals in the execution of tasks where their integrity and health may be at risk. As an example of this kind of training, we can cite the one associated with the firefighting. Among these studies were those carried out by Rahmalan et al. who used VR to instruct in estimating a fire [37], the one by Pitana et al. focused on training fire inspectors [38], or the study conducted by Wan for training inspectors of industrial oil deposits [15]. In line with the above studies, Li explored the use of VR for coal miners training [39]. Likewise, the authors of this paper have already introduced a new system to bring virtual reality closer to teachers [40], and have explored the use of a semi-assisted virtual experience creation system for training in the prevention of occupational risks [41]. After a thorough analysis of the state of the art, the following conclusions should be highlighted:

- Virtual Reality is a tool with a wide range of industrial applications.

- Virtual Reality can be successfully used for professional training so that they can act in situations of risk without affecting their health or physical integrity.

- The creation of all virtual experiences starts from scratch without using standard tools or framework, which implies cost overruns in the design phase.

- Existing solutions do not take into account the vulnerability of data that users expose in the system. Users in a virtual environment are providing information about themselves and how they interact, so these data must be properly protected.

\section{B. Mixed Reality}

As we saw in Fig. 1, another position of the "virtual continuum" is occupied by mixed reality (MR), whose characteristics are of interest to the object of this paper. The user in this region can perceive real and virtual objects, together but distinguishable from each other. Virtual objects must also interact with real ones. Also, the user has the ability to interact with these virtual objects in a natural way. For example, in a mixed reality scene that simulates the passage of objects through a real production chain, these objects must replicate real-world behavior and interact with both the user and the actuators in the assembly chain. Defining mixed reality is not an easy task, because of its constant evolution [42]. Milgran and Kishino defined it as "a mix of real and virtual objects within a single display." [5]. In 2019, Speicher et al. conducted a bibliographic review in order to provide a more specific definition of mixed reality, but their conclusion was that it "depends" [42]. Even if consensus has not been reached on the definition of MR, it is important to note that there is a difference from augmented reality (AR). Some authors describe the MR as an integration of VR and AR. In their case, Tepper et al. noted: "mixed reality merges many of the benefits of virtual reality and augmented reality" [43]. In other words, they offer the capabilities of a virtual world, where everything that happens is controlled by software, along with the user's perception of the real world. Analyzing the devices currently available on the market, the most commonly used is the Microsoft HoloLens device. Hololens is a Head Mounted Display (HMD) so it is placed on the head without the need to hold other devices on the hands. Version 2 is currently on the market. These devices offer a range of possibilities to define the experiences available to users. The main features of HoloLens are [44]: (1) head and eye tracking system; (2) microphone for voice commands; (3) accelerometers for the user's motion control, based on the acceleration of the head; (4) hand and finger tracking by computer vision and depth sensors. Also noteworthy are the actions that can be achieved by the use of hands. Microsoft Hololens has a very efficient gesture recognition system (e.g. hand closure, thumb grip and index finger, or select using index finger) (Fig. 3).

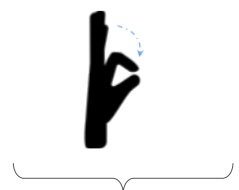

Grab/tap

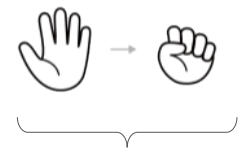

Close hand

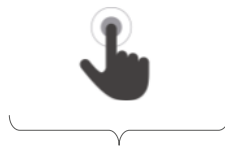

Touch
Fig. 3. HoloLens 2 gestures examples [45].

However, the HoloLens device also has limitations, as do any technology. For gestures, content creators should build virtual experiences according to the Hololens detection system, since hands can be hidden when using cameras and therefore their actions will not be detected. Research on virtual reality has accelerated the application of mixed reality in different cases of use. However, it is important not to consider that MR is simply an evolution or improvement of AR. This confusion may stem from the fact that the MR is a post-AR technology, but MR is really a technology that explores a different position from the "virtual continuum". Mixed reality makes digital information ubiquity possible, which has led to a significant increase in its application in the industry [46]. Previous research in augmented reality has facilitated the rapid reception of the MR. This is because some limitations of AR have been corrected by the new capacities provided by the MR [47]. The main applications of this technology in the industry support work at different points in the production chain. This support has mainly involved the incorporation of digital information into the execution of tasks [48]. Some of the most interesting applications are those related to the design of vehicles using aggregated information [49]. Another example is the adoption of the MR to offer operators a new user interface while working alongside robots or remotely with complex devices [50] [51]. It can be noted that significant efforts have been made in the scientific community to integrate the MR into the aviation industry, with particular emphasis on its airplane maintenance lines [52] [53] [54] [55] [56] [57]. The voice command interaction provided by the Hololens [58], together with the ability to provide information and designs at the operator's workplace, makes the MR a key tool for productivity improvement in the coming years [49] [59] [60], as well as for design, maintenance, security and quality control in the industry [48]. Likewise, the ability to interact and expose digital information in a real environment has made the MR a tool of relevance 
in the field of education and training in the industry [60]. The MR has been used in training to learn how to react in situations of risk [61] [62], or to provide a training environment for the performance of real tasks, greatly enriching the traditional training experience [63]. This improvement is seen in the new ways of training health care workers, taking advantage of the mixed reality both in tasks of special difficulty and in other everyday tasks such as stitching a patient [64]. These new VR and MR tools offer an opportunity to evolve educational and training methodologies [65]. Therefore, the MR is a tool that can be used in many areas such as industry, education, medicine, etc. The adoption of these new tools requires a proper process, since interaction with these elements may not be simple and therefore be rejected by users. For this reason, a process of user training must precede any action to implement a MR tool [48]. In addition, the degree of user acceptance will influence the success of the implementation of mixed reality tools as a support element in the execution of a task [59].

\section{Occupational Safety and Health}

The OSH is a important challenge that affect to the whole people around the world. In 2018 in EU27 3.1 million of the non-fatal accidents occurred, and 3.110 fatal accident, other study, conducted by Hämäläinen et al. reports that in 2014 the world saw 373 million accidents at work [66]. According to the study carried out by Takala, the number of deaths due to professional illness is 2 million and the number caused by an occupational accident is more than 300,000 [67]. The Global Burden of Disease Study of 2015 revealed that $5 \%$ of active people's mortality is due to occupational accidents or professional illnesses [68]. Without belittling the importance of protecting the human lives involved, a major component is the economic impact that the safety and health of workers can have on companies and, in general, on the national economy. According to the study by Buerau of Economic Analysis, the estimated cost of work-related accidents and professional illnesses is between $\$ 200-550$ billions [69]. These data reveal the important problem of workers' safety and health, which justifies the need for tools that help reduce these figures. These circumstances have led to significant increase in OSH research in recent years. Early research in this field comes from other areas, such as medicine [70], although it is already a research field in itself that is of great interest. Its relevance in terms of economic cost and human lives, has led us to analyse the relationships between employees' factors and their working environment that can determine the context for a potential occupational accident [71].

\section{Motivation AND Methodology}

From the study of the OSH situation in Europe and the world we can conclude that we can help the society with new tools and solutions to try to help the amount of people that could have accidents at work. The best way to help the workers is to provide them with the knowledge about how to avoid the accident or, if an accident occurred, how to get a safe him/herself and him/her colleagues. However, it is necessary to help the company with the best tools to train the whole workers team and do this training process like a easy, rapid and cheap way. Today, human augmentation is a technology with great capacity to apply in several user cases. From human augmentation, the authors have selected mixed reality technology as it offers a new way of interaction between the users and digital solution. With mixed reality a user can see a digital 3D object in the same point of view as the real environment, and if we use the HoloLens2 as HMD the user can see and interact with the $3 \mathrm{D}$ object thanks to the HoloLens 2 gesture and voice recognition. In the literature we find several different approaches of mixed reality with workers to help to do different tasks or help to learn several concepts or processes. So, the researchers create several mixed reality experiences for each paper. Thus, this is the same situation that slows down the application of the virtual reality application in engineering, construction and industry [14] [15] [16]. For this reason and using the literature review the authors introduce the TrainingMR. It is a solution to help the mixed reality application in the whole industry $4.0 \mathrm{OSH}$ prevention training process for any type of company. Furthermore, Training-MR helps to democratisation of the technology application because it reduces the cost and time spent by the entities to create, test and publish the mixed reality experiences.

\section{A. Methodology}

The creation of the Training-MR was carried out under an agile development method. The methodology chosen by the authors was Scrum. This methodology offers a great capacity to modify the objectives and tasks in a development team depending on the results that occur in each Sprint [72]. Scrum is a development methodology that works very well in research and development projects because the probability of unexpected events is very high as these are projects where uncertainty is important. Thus, development has been divided into two phases.

- Concept phase. The aim of this phase is to reduce the uncertainty of the project. Several proofs of concept $(\mathrm{PoC})$ have been carried out in order to assess whether the mixed reality technology was mature enough to be applied to the project. A laboratory test of the capabilities offered by mixed reality can be seen in Fig. 4. Here the authors check several proofs of concept to use MR as a tool to create MR experiences [73]. During this phase, the state-of-theart analysis of scientific advances related to the project was also carried out, and the whole requirements list was defined.

- Development phase. This corresponds to the important stage, during which different iterations of the work have been developed in scrum methodology in order to extend the features of Training-MR.

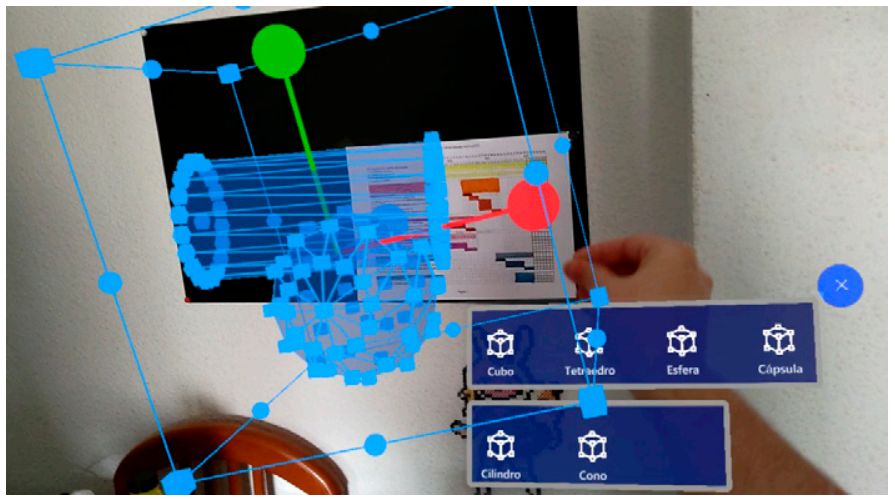

Fig. 4. PoC of the mixed reality capacity to interact with the user and virtual $3 \mathrm{D}$ object.

\section{TECHNICAL DESCRIPTION}

Focusing on the technical description, if we use a high level point of view then the Training-MR has 3 major modules working together. The modules are shown in Fig. 5 and are as follows:

- Editor: the module used to create the whole virtual experience. This module works as a plugin of Unity3D(https://unity.com/) to assist the creation process. Editor is composed of tools to render the scene, create a set of different kinds of components and conFig. them. The Editor is key to solving the cost problem since tools and functions are designed to reduce the time to create a MR experience. At the end of the creation process, the users can send the experience to the cloud for use in the training process.

- Cloud: it is the most important module when the mixed reality 
experiences are running. Cloud has the responsibility to manage the whole process in the experience. It is a communications hub that processes all events to determine if it is necessary to run a kind heavyweight algorithm.

- Glasses Client: this module runs in the user devices, and it is the first controller of virtual components behavior. The functions of the Glasses Client are: (1) render the scene, process common behaviour, (2) maintain synchronized event queue with the cloud to process all users actions (3) send all event data to the the Cloud, and (4) collect all data from the user experience to be sent to the cloud.
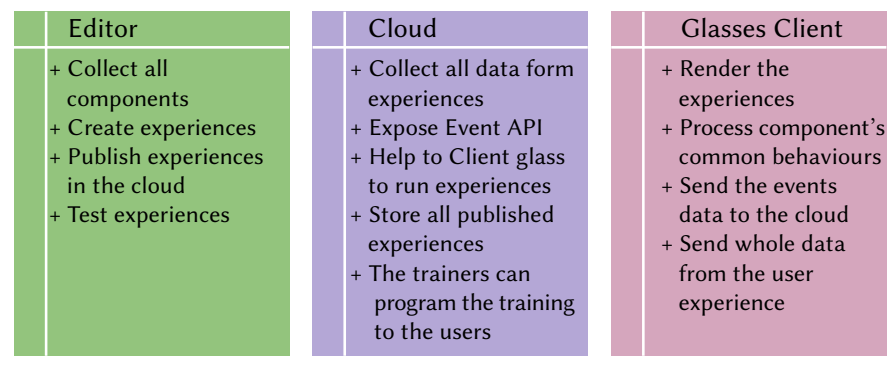

Fig. 5. Main requirement of the Training-MR modules.

However, the Editor module works isolated at the beginning of the workflow. Two kinds of important data can be highlighted in the workflow of the Training-MR: the virtual experience descriptor and the event descriptor. The first one is a high weight structure of data where any information can be found to create and run the virtual experience. The other one is a lightweight message between glasses and the cloud to process all actions in the MR experience. The virtual experience descriptor is introduced below, together with the event message in the "Communication issue" section.

\section{A. Virtual Experience Descriptor}

It corresponds to the core data of any experience in the TrainingMR and where information about any element in the virtual experience can be found. This descriptor is an attribute-value file in the JSON language. The most important parts in the descriptor are listing below:

- General data: data to describe the virtual experience, the most important property is the ID Virtual experience, needed to associate the running with the virtual experience in the cloud.

- Scenes descriptions: it constitutes a long list of the components in the catalog. The values of the whole properties of all components can be found here. The exception is the url to download the 3D assets used to render the elements.

- 3D Assets URL: the list of the urls to download 3D assets. These data are split off from the other properties for cybersecurity reasons.

Fig. 6 shows a fragment of the descriptor file. In this example the object "wear" and the parameters such as id, index, onValidSnapEvent, among others, are described.

\section{B. High Level of the Workflow}

In normal execution, the Editor does not participate in the run. The reason is that it is usually used to create the experience. Thus, the workflow starts in the glasses client when the user runs our application. The steps to be taken for the execution of the experience are described below and shown in the workflow diagram in the Fig. 7.

1. Start: at the beginning, the users wait for the experience in the hall. The hall is a welcome scene where the users can also interact with some dummy components. These components have been selected to help the user get familiar with the gestures, actions and behaviours from the components.

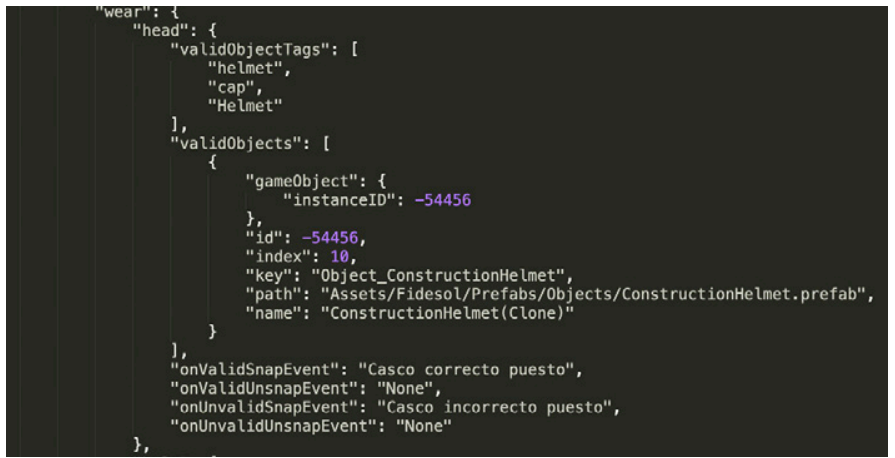

Fig. 6. Example of the mixed reality experience descriptor file.

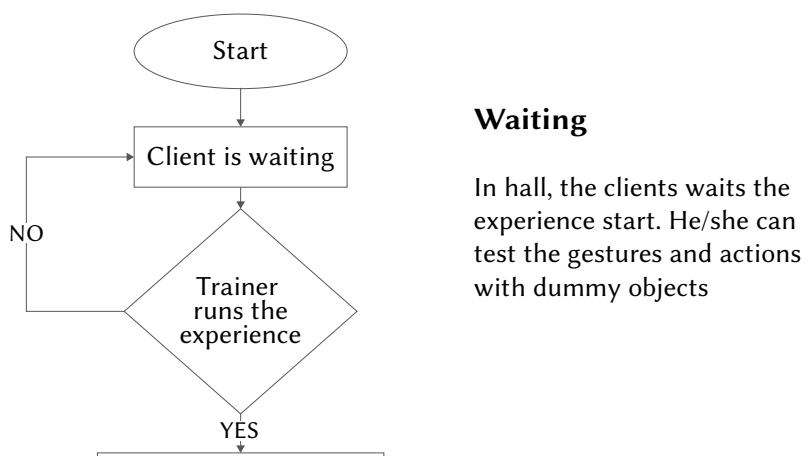

Download the

experience descriptor

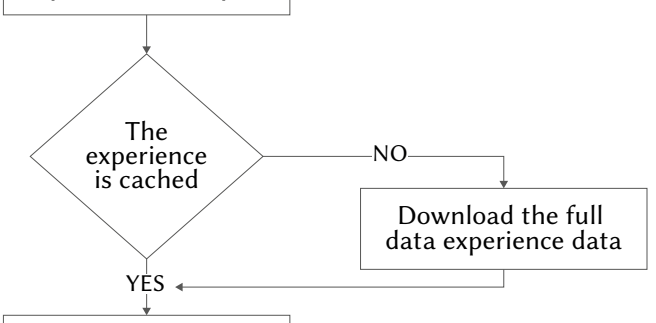

Full download

the $3 \mathrm{D}$ component
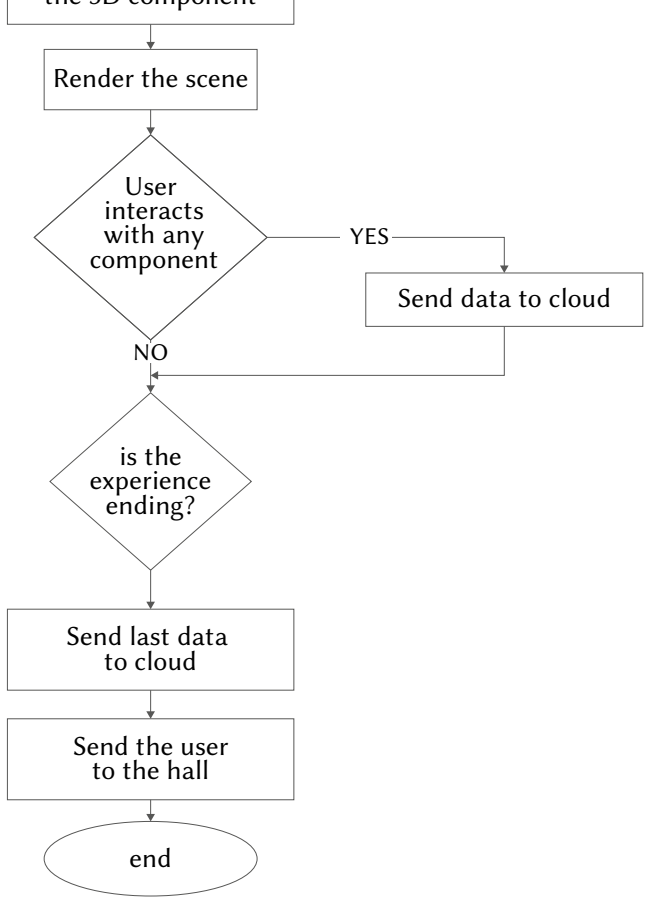

Fig. 7. High level of the Training-MR workflow. 
2. Load the experience: when the trainer has been selected by the cloud, the glasses client receives one message by web sockets. At this moment, the glasses client checks if the experience exists in the experiences cache then loads from the cache. If it is not cached, the app downloads the full description from the cloud.

3. Start the render process: the 3D engine runs the virtual experience, and prepares all logic subcomponents to be used in the execution, such as: event queue manager, communication abstraction layer, $3 \mathrm{D}$ components catalog and many others. Now the workflow is a list of events that have been triggered by the user. The glasses client runs different behaviours from the 3D components and sends several messages to the cloud.

4. End of the experience: this happens when the user successfully performs the last task. The glasses client runs the final actions to close the experience and moves the user to the hall, cleans the memory, sends data from the experience to the cloud, and performs other functions required to prepare the glasses client to run another experience.

\section{Training-MR Description}

At this point, once the virtual experience descriptor is known, it is needed to describe the Training-MR in more detail. This description is faced by differentiating the following parts: (1st) the cloud and the glasses are described together to facilitate their understanding given their interconnection, (2nd) we introduce the Editor and how it works (3rd) finally, the communication section exposes how the client and cloud share information, and the process by which data are protected from cyberattacks.

\section{The Cloud and Glasses Client}

It is important to analyse together the Cloud and the Glasses Client, since the design decisions of both are interconnected. Therefore, in a common case of use, a large number of requests are sent to the Cloud from all the glasses clients that are running at the same time. In this way, the microservices architecture in the Cloud is selected Microservices architecture allows the rapid scale of services in peak requests, so the platform automatically reduces these services when the number of requests returns to normal. Services have been designed with Stateless Design Pattern [74], so they are run in an isolated way and endpoints of the public API have been designed to solicit whole data to process. Furthermore, in our solution there are no links or relationships between two services. The technology used to scale services is Docker. On the other hand, the main workflow is the one that takes place in the Glass Client. The Cloud is an unlimited resource to send and request data, from Glass Client's point of view. The Glasses Client workflow acts as an action dispatcher. When an input is detected (for example, user actions), the Glasses Client processes the action according to its code. For this reason the Glasses Client is an Event-driven Architecture solution [75]. The Event-driven
Architecture focuses the workflow on event processing. An event could appear for several causes, in our case, the user will be the most important event generator, moreover events from the cloud can also occur. The most important code component in Glasses Client is the Event Queue, where the events are waiting to be processed. Not all events are considered in the same way, so user events are priorities because their delay could cause the freeze or user view error.

The Glasses Client works as a Thin Client in our scheme, so the user could interact with any component that the glasses has renderized. The 3D components present a behaviour similar to the sequence diagram shown in Fig. 8. The sequence starts when the user interacts with the component, which provoques the invocation of the EventManager. The EventManager has the responsibility to start the communication process with the Cloud and invoque de virtual component to modify its properties. Once the response from the Cloud is received by the glasses, virtual component invocation occurs. The properties of the virtual component could be modified in two ways. The first one through a simple action such as launching or moving an object, etc. The second way allows the modification of virtual components with the result of the heavyweight algorithms from the Cloud.

\section{Communication Issues}

The presented workflow between the Cloud and the Glasses Client must deal with a large number of requests. The Cloud has a Restful API pattern programmed with JSON language. Restful API is a lightweight API standard in Internet services. The TrainingMR has a lot of endpoints to manage all information such as User, Student, or Experience, among others although the most important is the ExerciseEvent. The responsibility of the ExerciseEvent is to manage all events for the experiences. However, the API Rest does not handle all communications. When one experience has started, a special message is sent to the Cloud in order to create one web socket between the Cloud and the Glasses Client. The web socket is a channel used by the Cloud to communicate asynchronous data to the Glasses Client. For example, when a heavyweight algorithm process has finished the result should be sent to the Glasses Client that has invoked the algorithm.

Event message An event message is sent to the Cloud caused by a certain trigger or behaviour. The events are usually triggered by the glasses to the Cloud, but there can also be events created and triggered by the Cloud. An example of these events are those from the trainers (actions such as force stop or communication). The Event message has been designed with a short and simple structure.This decision is based on a design that ensures fast message processing. There are different types of messages, but they all have the same properties as the following:

- Type: Type Event.

- ID Virtual Experience: used by the Cloud to identify the virtual

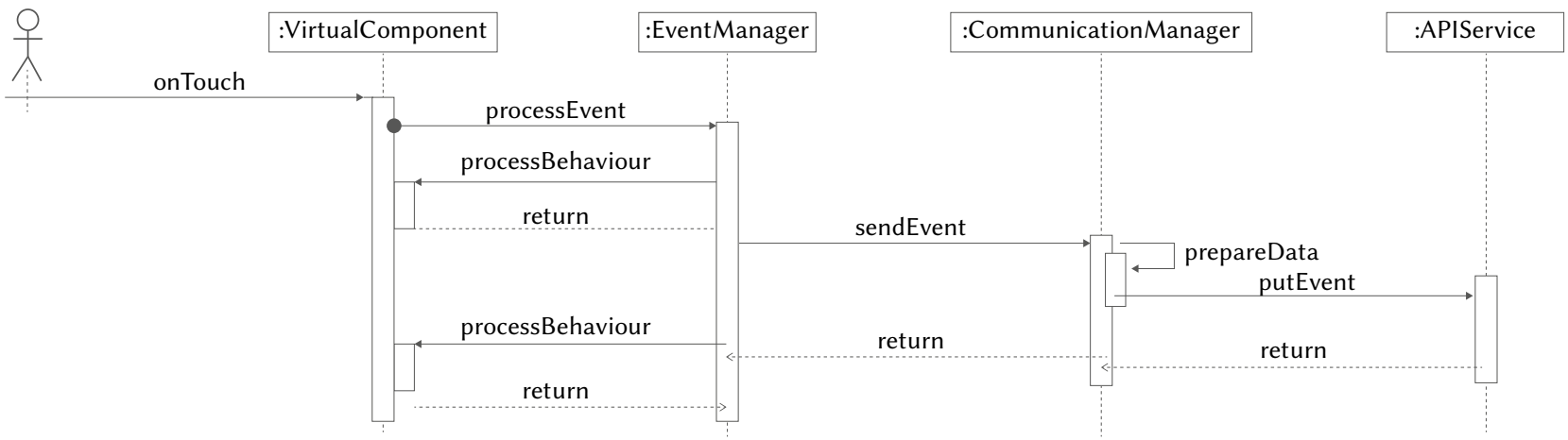

Fig. 8. Sequence diagram of Glasses Client and Cloud. 
experience.

- ID running: used by the Cloud to identify a particular execution in any glasses.

- ID user: user running the experience.

- timestamp: timestamp from the glasses.

- time: run time measured in milliseconds. Zero corresponds to the beginning of the MR experience.

Some of the Event messages properties can be customized, which are collected in a list of key-values. Data from the experience are processed in the Cloud to determine whether it is necessary to run a heavyweight algorithm, although all data are always collected and stored in a database. The database selected in the Training-MR is NoSQL instead of the traditional SQL, due to the need for flexibility to store different data sets together and the unknown structure of data from future Event messages. These data will be of particular relevance for analysis in order to know how users interact with mixed reality.

\section{Cybersecurity Issues}

Cybersecurity is currently a must-have feature in any software or ICT platform, due to the increase in the value of data in recent years. Two types of data coexist in our platform: virtual reality description data, and data related to user interaction in the virtual experience. The first group can contain information from a company, being the end point at which a hacker could steal technical information, for example the end point where a hacker could download a 3D asset and in this way, he/she could steal the technical information. The second group is the data about how the users interact in the experience, this data set could be analysed to determine a lot of information about the industrial process on which the training was designed. To protect these data the solution created has:

- API Key: API requests have parameters about the code that did the request, this is the API KEY, a unique application identifier (code) and it is hardcore in the code so, allows the block of all requests when a cybersecurity attack has occurred.

- Encrypted communication end to end: Communications use SSL encryption for data protection when transmitted over the Internet. In this way, the information will not be understood, if any hacker attempts to sniff the network traffic.

- Encrypted store: Data are encrypted before being stored in the NoSQL database. Moreover, when the glasses caches the data for future use, the virtual experience descriptor is encrypted also.

\section{The Editor}

The Editor is a module that works isolated from the entire solution. The main objective of the Editor is to provide a toolbox to help content creators. Key components are listed below:

- 3D Viewer. It is the most important requirement because the creator needs to design a 3D scene that will be rendered by the MR glasses. The user selects components from the virtual components catalog to be assigned to the 3D scene. The user must set the properties of these 3D components, such as, physical properties and simple actions (touch, grip, push, etc), among others. At the end of the process, the creator gets the virtual scene with all items positioned on the 3D scene, and all the 3D components completely parameterized.

- 3D Catalog. The 3D Catalog and the 3D Viewer work together. The catalog is a powerful generic 3D component search tool to be used for creating scenes. The $3 \mathrm{D}$ components that appear in this catalog are the high abstraction of the tools, situations or triggers.

- -Scene tester. Test scenes are fundamental in the creation process. For example, the creator might need to test the different settings in the scene or probe the relationship between two objects. The Editor allows the creator to test the scenes quickly and easily.

- Publish the mixed reality experience. The Editor and the Cloud are linked. At the end of the process, the creator will upload the mixed reality training to the Cloud. This action will not be available to all users, but only trainers selected by the creator will be able to access this new MR experience.

In order to provide an example of how the Editor works, its application is exposed for selecting the correct electrical wire with an alligator clip. In Fig. 9, a scheme of the proposed virtual scene is displayed. The creator has to describe: the alligator clip, the electrical wires and the triggers (one right option and two wrong). It is important to highlight that these items should be selected by the creator from the Catalog: the physical object for the electrical wires, a hand object to abstract the alligator clip that will be grabbed by the user, and the trigger zone attached to the electrical wires (represented in the scheme by the blue items around the electrical wires). The electrical wires and the alligator clip are physical objects, so the creator has to set their physical properties such as weight, and 3D assets, etc. The triggers are components that do not have 3D assets, weight or physical behaviour, but require a special event when the area is touched by the alligator clip. This contact does not cause movements but the test reaction, to identify whether the action has been successful or not.

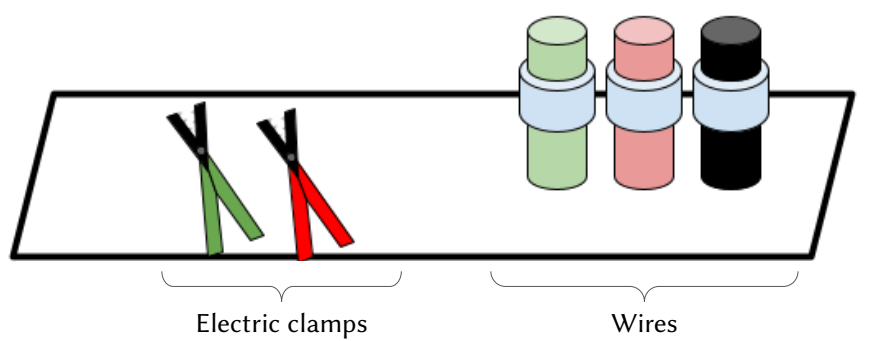

Fig. 9. Example schema of the MR virtual scene example.

Finally, a simple example is provided to describe the workflow of the Editor, although it allows the creator to compose complex behaviours and trigger hierarchical events. Fig. 10 shows the definition of the workflow from a real virtual scene in which the trigger identifies as a failure the lack of worker protection equipment in a welding training.

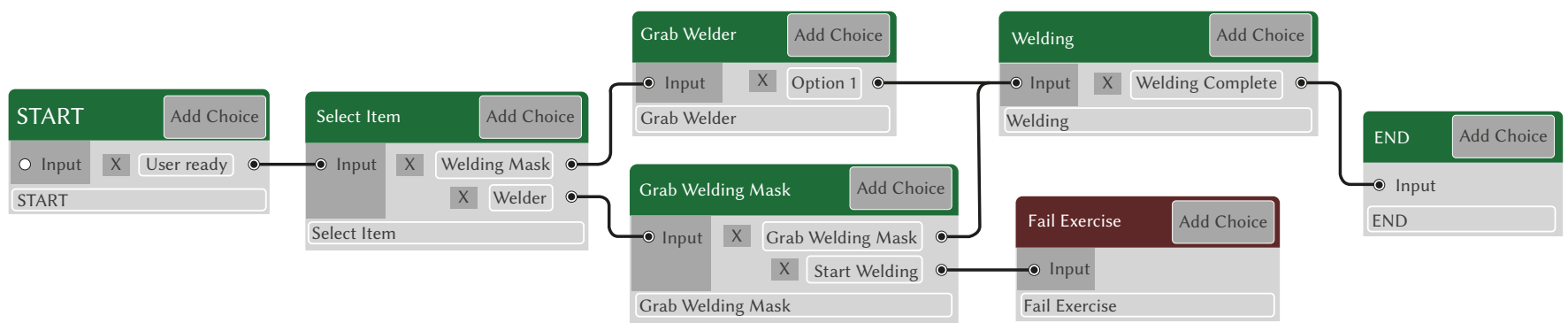

Fig. 10. Example the trigger and behaviour composition in the Editor. 
TABLE I. RESUME OF THE MR/VR SOLUTIONS

\begin{tabular}{ccccc}
\hline Feature & Training - MR & Blufamsterdam technology & Neurodigital tech & ClassVR \\
\hline Mixed reality technology & OK & OK & NO & NO \\
\hline Creation content toolkit & OK & NO SDK & NO & NO \\
\hline Publication capacity & OK & NO & Without details & OK \\
\hline Cyber security design & OK & Without details & N \\
\hline
\end{tabular}

Therefore, the great potential of the Editor makes it possible for users to quickly and easily create mixed reality experiences. Moreover, it is necessary to improve the speed to create MR experience because the economical cost is a high stopper to apply MR as a tool, thus the Training-MR helps to improve training especially in occupational health and safety by not compromising the integrity of the workers.

\section{Discussion and Conclusion}

Virtual reality and mixed reality correspond to new technologies that can be adopted in the productive processes of companies belonging to different sectors of activity. Researchers have tried to develop solutions that apply augmented reality to solve problems in different fields such as education, industry, engineering, and so on. Mixed reality allows us to go one step further. MR is a powerful tool to improve user training and education as they can interact with both digital and real components at the same time. However, the adoption of MR as a training tool presents the same problems identified for the application of virtual reality [14] [15] [16]. These are the high costs and excessive time required for implementation. The solution to create, test, and publish MR experiences presented in this paper. Training-MR is a solution to all of the problems mentioned above. The main advantages achieved with this tool are listed below:

- From the user perspective, the MR experience is a service from the Cloud. The Glasses Client is a dummy application that only detects the user actions to send requests to the Cloud and process the response data.

- The architecture has been designed to be scalable based on the number of the requests. Therefore, the Cloud can respond to any number of Glasses Clients worldwide.

- The Glasses Client has been designed to prioritize the reaction of user actions to avoid the freezing effect. The Event-driven Architecture enables the prioritization of user events, queuing the least relevant events.

- Training-MR has been built on the principles of cybersecurity and best practices applied to information security. Cybersecurity requirements have been addressed integrated into the platform considering their efficiency and optimization, resulting in a fully secure platform.

- Training-MR, with its Editor, allows to the user to create easy, quickly and quality mixed reality experiences and test these to fix problems o improve the scenes

- A user can share with others the MR experiences created by Training-MR. Training-MR offers the capability to publish the MR experience in the Cloud, and these experiences can be used by other users. In this way, it helps to the democratization of the MR

- The MR experiences have been loaded into the Glasses Client in a quick and easy way. Users can interact with MR experience and improve their knowledge of occupational safety and health.

We compare Training-MR with other public solutions to train the OSH prevention process. Nowadays, the most common situation is that the entities with know-how of the OSH training or experience in virtual reality solutions do not have mixed reality solutions. The most important features to help the easy and rapid application of the mixed reality solutions are used to compare the solutions. These features are: capacity to help to create experiences, mixed reality experiences, experience public capability, cybersecurity design. The Table I resumes our review with other products. It is not common to find solutions with MR technology and usually are for entertainment business. Virtual reality for education and training proposals is more common. Other products are only a set of VR experiences of several kinds of topics. This is the ClassVR case. Rarely find solutions to create content with tools from VR technology owner, and the tools are a SDK to create content by code develop. The public information about the solutions does not explain any cyber security issues.

Our platform is currently in the laboratory testing phase. This phase has taken place after the research team has conducted certain relevant tests, from which a set of MR experiences have been created and will be tested by control users. These users will select an experience, choosing between virtual reality or augmented reality, depending on their knowledge of each technology. After this test with the control users, a final phase called "pilot experience" will be created. In this phase the platform will be tested for training common users in a set of mixed reality controllers. Despite the platform's advantages, this study is not without limitations:

- Gestures. Hololens2 is the most powerful MR device but has a limitation in terms of gesture recognition. It is not possible to recognize a movement that happens behind the user, just as it does not detect an object that is covered by another.

- Multiplayer experience. The platform currently does not allow the creation of a multiplayer MR experience. In the future, special attention will be given to collaborative training.

- Teacher assistance. This has close relationships with the multiplayer. The platform does not currently allow to introduce the teacher's actions into the MR experience as inany learning process in which the teacher interacts with the student.

- Real test. Once the pilot experience is completed, it would be of great importance to test the platform in an industrial environment. To test the components, behaviours, and results of the training in occupational safety and health to validate the MR as a powerful tool for this learning.

- Data analysis. Our platform generates a huge set of data for every MR experience. These data are of great value because their analysis will allow to establish relationships between variables specific to workers with the conditions of the workplace and the use of tools, in order to determine the situations that could lead to an occupational accident or a professional illness.

\section{ACKNOWLEDGMENT}

The whole Fidesol team for the great work and capacity in "Interacción y Programación Inteligente para la Industria 4.0 a través de la Realidad Mixta y Fabricación Aditiva" project with code "PY18RE-0024". 
REFERENCES

[1] A. P. Botha, "Rapidly arriving futures: Future readiness for industry 4.0," South African fournal of Industrial Engineering, vol. 29, nov 2018, doi: 10.7166/29-3-2056

[2] J. A. Saucedo-Martínez, M. Pérez-Lara, J. A. Marmolejo-Saucedo, T. E. Salais-Fierro, P. Vasant, "Industry 4.0 framework for management and operations: a review," Journal of Ambient Intelligence and Humanized Computing, vol. 9, pp. 789-801, jun 2017, doi: 10.1007/s12652-017-0533-1.

[3] J. Sengupta, S. Ruj, S. D. Bit, "A secure fog- based architecture for industrial internet of things and industry 4.0," IEEE Transactions on Industrial Informatics, vol. 17, pp. 2316-2324, apr 2021, doi: 10.1109/ tii.2020.2998105.

[4] J. L. Ruiz-Real, J. Uribe-Toril, J. A. Torres, J. D. Pablo, "Artificial Intelligence in Business and Economics Research: Trends and Future," Journal of Business Economics and Management, vol. 22, pp. 98-117, oct 2020, doi: 10.3846/jbem.2020.13641.

[5] P. Milgram, F. Kishino, "A taxonomy of mixed reality visual displays," IEICE Trans. Information Systems, no. 12, pp. 1321-1329, 1994.

[6] M.-D. González-Zamar, E. Abad-Segura, "Implications of virtual reality in arts education: Research analysis in the context of higher education," Education Sciences, vol. 10, p. 225, aug 2020, doi: 10.3390/educsci10090225.

[7] J. Wolfartsberger, "Analyzing the potential of virtual reality for engineering design review," Automation in Construction, vol. 104, pp. 27-37, aug 2019, doi: 10.1016/j.autcon.2019.03.018.

[8] J. M. Lombardo, M. A. Lopez, M. López, M. León, F. Miron, J. Arambarri, D. Álvarez, "MOBEEZE. natural interaction technologies, virtual reality and artificial intelligence for gait disorders analysis and rehabilitation in patients with parkinson's disease," International fournal of Interactive Multimedia and Artificial Intelligence, vol. 5, no. 6, p. 54, 2019, doi: 10.9781/ijimai.2019.07.003.

[9] Y.-C. Du, S.-C. Fan, L.-C. Yang, "The impact of multi-person virtual reality competitive learning on anatomy education: a randomized controlled study," BMC Medical Education, vol. 20, oct 2020, doi: 10.1186/s12909-02002155-9.

[10] D. V. Joao, P. Z. Lodetti, A. B. dos Santos, M. A. I. Martins, S. de Francisci, J. F. B. Almeida, "Augmented reality application to assist in on-field activities on the electrical sector," in 2021 IEEE Power \& Energy Society Innovative Smart Grid Technologies Conference (ISGT), feb 2021, IEEE.

[11] Y. Dan, Z. Shen, Y. Zhu, L. Huang, "Using mixed reality (MR) to improve on-site design experience in community planning," Applied Sciences, vol. 11, p. 3071, mar 2021, doi: 10.3390/app11073071

[12] E. Bottani, F. Longo, L. Nicoletti, A. Padovano, G. P. C. Tancredi, L. Tebaldi, M. Vetrano, G. Vignali, "Wearable and interactive mixed reality solutions for fault diagnosis and assistance in manufacturing systems: Implementation and testing in an aseptic bottling line," Computers in Industry, vol. 128, p. 103429, jun 2021, doi: 10.1016/j.compind.2021.103429.

[13] "Employer-reported workplace injuries and illnesses 2016," Bureau of labor statistics. U.S Departament of labor, 2016.

[14] J. M. D. Delgado, L. Oyedele, P. Demian, T. Beach, "A research agenda for augmented and virtual reality in architecture, engineering and construction," Advanced Engineering Informatics, vol. 45, p. 101122, aug 2020, doi: 10.1016/j.aei.2020.101122.

[15] J. Wan, Y. Zheng, Y. Li, H. Mei, L. Lin, L. Kuang, "Oil depot safety inspection and emergency training system based on virtual reality technology," IOP Conference Series: Materials Science and Engineering, vol. 782, p. 042018, apr 2020, doi: 10.1088/1757- 899x/782/4/042018.

[16] J. S. D. Orlean G. Dela Cruz, "Virtual reality (vr): A review on its application in construction safety," Turkish fournal of Computer and Mathematics Education, vol. 12, no. 11, pp. 3379-3393, 2021.

[17] R. R. Nick Bostrom, "Ethical issues in human enhancement," New Waves in Applied Ethics, pp. 120- 152, 2008.

[18] T. Garcia, R. Sandler, "Enhancing justice?," NanoEthics, vol. 2, pp. 277287, nov 2008, doi: 10.1007/s11569-008- 0048-5.

[19] Z. Li, "Ethical problems concerning human augmentation technology and its future aspects," in Proceedings of the 7th International Conference on Humanities and Social Science Research (ICHSSR 2021), 2021, Atlantis Press.

[20] R. Raisamo, I. Rakkolainen, P. Majaranta, K. Salminen, J. Rantala, A. Farooq, "Human augmentation: Past, present and future," International
Journal of Human- Computer Studies, vol. 131, pp. 131-143, nov 2019, doi: 10.1016/j.ijhcs.2019.05.008.

[21] E. Pietrafesa, S. Iavicoli, A. Martini, R. Simeone, Polimeni, "Occupational safety and health education and training: an innovative format and experience," in 6th International Conference on Higher Education Advances (HEAd'20), jun 2020, Universitat Politècnica de València.

[22] P.-E. Boileau, "Sustainability and prevention in occupational health and safety," Industrial Health, vol. 54, no. 4, pp. 293-295, 2016, doi: 10.2486/ indhealth.54-293.

[23] A. Simeone, A. Caggiano, L. Boun, R. Grant, "Cloud- based platform for intelligent healthcare monitoring and risk prevention in hazardous manufacturing contexts," Procedia CIRP, vol. 99, pp. 50-56, 2021, doi: 10.1016/j.procir.2021.03.009.

[24] R. Suganya, S. Gowtham, "Individual health and safety monitoring of workers in deep underground mines using IOT", fournal of Physics: Conference Series, vol. 1717, p. 012044, jan 2021, doi: 10.1088/17426596/1717/1/012044.

[25] M. B. V. kumar, M. B. Jayasree, M. D. Kiruthika, "Iot based underground coalmine safety system," fournal of Physics: Conference Series, vol. 1717, p. 012030, jan 2021, doi: 10.1088/1742-6596/1717/1/012030.

[26] V. Jayasree, M. N. Kumari, "IOT based smart helmet for construction workers," in 2020 7th International Conference on Smart Structures and Systems (ICSSS), jul 2020, IEEE.

[27] I. Campero-Jurado, S. Márquez-Sánchez, J. Quintanar- Gómez, S. Rodríguez, J. M. Corchado, "Smart helmet 5.0 for industrial internet of things using artificial intelligence," Sensors, vol. 20, p. 6241, nov 2020, doi: 10.3390/s20216241.

[28] M. A. Gigante, "Virtual reality: Definitions, history and applications," in Virtual Reality Systems, Elsevier, 1993, pp. 3-14, doi: 10.1016/b978-0-12227748-1.50009-3.

[29] S. S. Kardong-Edgren, S. L. Farra, G. Alinier, H. M. Young, "A call to unify definitions of virtual reality," Clinical Simulation in Nursing, vol. 31, pp. 28-34, jun 2019, doi: 10.1016/j.ecns.2019.02.006.

[30] F. Quigley, A. Moorhead, R. Bond, H. Zheng, T. McAloon, "A virtual reality training tool to improve weight-related communication across healthcare settings," in Proceedings of the 31st European Conference on Cognitive Ergonomics, sep 2019, ACM.

[31] E. A.-L. Lee, K. W. Wong, C. C. Fung, "How does desktop virtual reality enhance learning outcomes? a structural equation modeling approach," Computers \& Education, vol. 55, pp. 1424-1442, dec 2010, doi: 10.1016/j. compedu.2010.06.006.

[32] C. A. Cohen, M. Hegarty, "Visualizing cross sections: Training spatial thinking using interactive animations and virtual objects," Learning and Individual Differences, vol. 33, pp. 63-71, jul 2014, doi: 10.1016/j. lindif.2014.04.002.

[33] N. Bouali, E. Nygren, S. S. Oyelere, J. Suhonen, V. Cavalli-Sforza, "Imikode," in Proceedings of the 19th Koli Calling International Conference on Computing Education Research, nov 2019, ACM.

[34] S. Greenwald, A. Kulik, A. Kunert, S. Beck, B. Frohlich, S. Cobb et al., Technology and applications for collaborative learning in virtual reality. 2017.

[35] T. Civelek, E. Ucar, H. Ustunel, M. K. Aydı, "Effects of a haptic augmented simulation on k-12 students' achievement and their attitudes towards physics," EURASIA fournal of Mathematics, Science and Technology Education, vol. 10, dec 2014, doi: 10.12973/eurasia.2014.1122a.

[36] C. Porter, J. Smith, E. Stagar, A. Simmons, M. Nieberding, C. Orban, J. Brown, A. Ayers, "Using virtual reality in electrostatics instruction: The impact of training," Physical Review Physics Education Research, vol. 16, sep 2020, doi: 10.1103/physrevphyseducres.16.020119.

[37] R. H., "Development of virtual reality training for fire safety education," International fournal of Advanced Trends in Computer Science and Engineering, vol. 9, pp. 5906-5912, aug 2020, doi: 10.30534/ ijatcse/2020/253942020.

[38] T. Pitana, H. Prastowo, A. P. Mahdali, "The development of fire safety appliances inspection training using virtual reality (VR) technology," IOP Conference Series: Earth and Environmental Science, vol. 557, p. 012064, sep 2020, doi: 10.1088/1755- 1315/557/1/012064.

[39] M. Li, Z. Sun, Z. Jiang, Z. Tan, J. Chen, "A virtual reality platform for safety training in coal mines with AI and cloud computing," Discrete Dynamics in Nature and Society, vol. 2020, pp. 1-7, oct 2020, doi: 
$10.1155 / 2020 / 6243085$

[40] J. M. Lombardo, M. A. Lopez, V. García, M. López, R. Cañadas, S. Velasco, M. León, "PRACTICA. a virtual reality platform for specialized training oriented to improve the productivity," International fournal of Interactive Multimedia and Artificial Intelligence, vol. 5, no. 4, p. 94, 2019, doi: 10.9781/ijimai.2018.04.007.

[41] M. A. Lopez, J. M. Lombardo, R. González-Crespo, "Educon 2021-creame: human augmentation platform for thecreation of training in educational lakes inherent todangerous situations." 2021.

[42] M. Speicher, B. D. Hall, M. Nebeling, "What is mixed reality?" in Proceedings of the 2019 CHI Conference on Human Factors in Computing Systems, may 2019, ACM.

[43] O. M. Tepper, H. L. Rudy, A. Lefkowitz, K. A. Weimer, S. M. Marks, C. S. Stern, E. S. Garfein, "Mixed reality with HoloLens," Plastic and Reconstructive Surgery, vol. 140, pp. 1066-1070, nov 2017, doi: 10.1097/ prs.0000000000003802.

[44] Microsoft, "Hololens 2 techspec," [Online]. Available: ht tp s:// www.microsoft.com/en-us/d/hololens 2/91pnzzznzwcp?activetab=pivot:techspecstab.

[45] Microsoft, "Hololens 2 gestures for authoring and navigating in dynamics 365 guides," [Online]. Available: https://docs.microsoft.com/en- us/ dynamics365/mixed-reality/guides/authoring- gestures-hl2.

[46] S. Rokhsaritalemi, A. Sadeghi-Niaraki, S.-M. Choi, "A review on mixed reality: Current trends, challenges and prospects," Applied Sciences, vol. 10, p. 636, jan 2020, doi: 10.3390/app10020636.

[47] R. G. Boboc, F. Gîrbacia, E. V. Butilă, "The application of augmented reality in the automotive industry: A systematic literature review," Applied Sciences, vol. 10, p. 4259, jun 2020, doi: 10.3390/app10124259.

[48] W. Kurschl, S. Pimminger, J. Schönböck, M. Augstein, J. Altmann, "Using mixed reality in intralogistics - are we ready yet?," Procedia Computer Science, vol. 180, pp. 132-141, 2021, doi: 10.1016/j.procs.2021.01.136.

[49] A. Kaluza, M. Juraschek, L. Büth, F. Cerdas, C. Herrmann, "Implementing mixed reality in automotive life cycle engineering: A visual analytics based approach," Procedia CIRP, vol. 80, pp. 717-722, 2019, doi: 10.1016/j. procir.2019.01.078.

[50] B. Bejczy, R. Bozyil, E. Vaičekauskas, S. B. K. Petersen, S. Bøgh, S. S. Hjorth, E. B. Hansen, "Mixed reality interface for improving mobile manipulator teleoperation in contamination critical applications," Procedia Manufacturing, vol. 51, pp. 620-626, 2020, doi: 10.1016/j. promfg.2020.10.087.

[51] R. Zhang, X. Liu, J. Shuai, L. Zheng, "Collaborative robot and mixed reality assisted microgravity assembly for large space mechanism," Procedia Manufacturing, vol. 51, pp. 38-45, 2020, doi: 10.1016/j.promfg.2020.10.007.

[52] A. Siyaev, G.-S. Jo, "Towards aircraft maintenance metaverse using speech interactions with virtual objects in mixed reality," Sensors, vol. 21, p. 2066, mar 2021, doi: 10.3390/s21062066.

[53] X. Wang, "Editorial visualization in engineering," Visualization in Engineering, vol. 2, mar 2014, doi: 10.1186/2213-7459-2-1.

[54] H. Silva, R. Resende, M. Breternitz, "Mixed reality application to support infrastructure maintenance," in 2018 International Young Engineers Forum (YEF-ECE), may 2018, IEEE.

[55] H. Eschen, T. Kötter, R. Rodeck, M. Harnisch, T. Schüppstuhl, “Augmented and virtual reality for inspection and maintenance processes in the aviation industry," Procedia Manufacturing, vol. 19, pp. 156-163, 2018, doi: 10.1016/j.promfg.2018.01.022.

[56] A. Fonnet, N. Alves, N. Sousa, M. Guevara, L. Magalhaes, "Heritage BIM integration with mixed reality for building preventive maintenance," in $201724^{\circ}$ Encontro Português de Computação Gráfica e Interação (EPCGI), oct 2017, IEEE.

[57] J. Christian, H. Krieger, A. Holzinger, R. Behringer, "Virtual and mixed reality interfaces for e- training: Examples of applications in light aircraft maintenance," in Lecture Notes in Computer Science, Springer Berlin Heidelberg, 2007, pp. 520-529.

[58] F. D. Pace, F. Manuri, A. Sanna, D. Zappia, "A comparison between two different approaches for a collaborative mixed-virtual environment in industrial maintenance," Frontiers in Robotics and AI, vol. 6, mar 2019, doi: 10.3389/frobt.2019.00018.

[59] S. R. Sorko, C. Trattner, J. Komar, "Implementing AR/MR - learning factories as protected learning space to rise the acceptance for mixed and augmented reality devices in production," Procedia Manufacturing, vol.
45, pp. 367-372, 2020, doi: 10.1016/j.promfg.2020.04.037.

[60] S. Lang, M. S. S. D. Kota, D. Weigert, F. Behrendt, "Mixed reality in production and logistics: Discussing the application potentials of microsoft HoloLensTM," Procedia Computer Science, vol. 149, pp. 118-129, 2019, doi: 10.1016/j.procs.2019.01.115

[61] L. Wunder, N. A. G. Gomez, J. E. Gonzalez, G. Mitzova- Vladinov, M. Cacchione, J. Mato, C. L. Foronda, J. A. Groom, "Fire in the operating room: Use of mixed reality simulation with nurse anesthesia students," Informatics, vol. 7, p. 40, sep 2020, doi: 10.3390/informatics7040040.

[62] H. F. Moore, M. Gheisari, "A review of virtual and mixed reality applications in construction safety literature," Safety, vol. 5, p. 51, aug 2019, doi: 10.3390/safety5030051.

[63] M. Czarski, Y. T. Ng, M. Vogt, M. Juraschek, B. Thiede, P. S. Tan, S. Thiede, C. Herrmann, "A mixed reality application for studying the improvement of HVAC systems in learning factories," Procedia Manufacturing, vol. 45, pp. 373-378, 2020, doi: 10.1016/j.promfg.2020.04.039.

[64] A. Rojo, L. Raya, A. Sanchez, "A novel mixed reality solution based on learning environment for sutures in minor surgery," Applied Sciences, vol. 11, p. 2335, mar 2021, doi: 10.3390/app11052335.

[65] K. Kounlaxay, S. K. Kim, "Design of learning media in mixed reality for lao education," Computers, Materials \& Continua, vol. 64, no. 1, pp. 161180, 2020, doi: 10.32604/cmc.2020.09930.

[66] J. T. T. B. K. Päivi Hämäläinen, "Global estimates of occupational accidentsand workrelated illnesses 2017," in World Congress on Safety and Health at Work 2017, 3-4 September 2017 Singapore, 2017.

[67] J. Takala, P. Hämäläinen, K. L. Saarela, L. Y. Yun, K. Manickam, T. W. Jin, P. Heng, C. Tjong, L. G. Kheng, S. Lim, G. S. Lin, "Global estimates of the burden of injury and illness at work in 2012," fournal of Occupational and Environmental Hygiene, vol. 11, pp. 326-337, apr 2014, doi: 10.1080/15459624.2013.863131.

[68] H. Wang, M. Naghavi, C. Allen, R. M. Barber, "Global, regional, and national life expectancy, all- cause mortality, and cause-specific mortality for 249 causes of death, 1980-2015: a systematic analysis for the global burden of disease study 2015," Lancet, vol. 388, no. 10053, pp. 1459-1544., 2016.

[69] "Employer-reported workplace injury and illness - 2016," Bureau of Labor Statistics, 2017. [Online]. Available: https://www.bls.gov/news. release/pdf/ osh.pdf.

[70] D. Fan, C. J. Zhu, A. R. Timming, Y. Su, X. Huang, Y. Lu, "Using the past to map out the future of occupational health and safety research: where do we go from here?", The International fournal of Human Resource Management, vol. 31, pp. 90-127, sep 2019, doi: 10.1080/09585192.2019.1657167.

[71] A. Kramer, S. Cho, R. S. Gajendran, "12-year longitudinal study linking within-person changes in work and family transitions and workplace injury risk," Journal of Safety Research, vol. 75, pp. 140-149, dec 2020, doi: 10.1016/j.jsr.2020.08.009.

[72] K. Schwaber, "SCRUM development process," in Business Object Design and Implementation, Springer London, 1997, pp. 117-134.

[73] M. A. Lopez, M. D. Ruiz, D. Alvarez, "Designme-mr: Toolbox for the creation of learning scenes to training in occupation risk prevention with mixed reality." June 2021.

[74] L.-P. T. Kamalmeet Singh, Adrian Ianculescu, Design Patterns and Best Practices in Fava. 2018.

[75] M. Richards, Software Architecture Patterns. 2015.

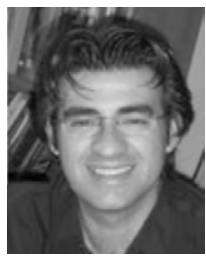

\section{Miguel Angel López}

He was graduated in Technical Engineering in Computer Systems from University of Almería, and in Computer Engineering and Master's Degree in Soft Computing and Intelligent Systems from University of Granada. At the moment, he is the CTO at Fidesol where he performs different roles. He is currently a $\mathrm{PhD}$ student at International University of La Rioja (UNIR). His research focuses on distributed systems, management, integration and analysis of data, robotics, fuzzy logic systems, human augmented, and the development of virtual/mixed reality environments. 


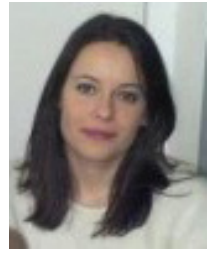

\section{Sara Terrón}

$\mathrm{PhD}$ in Business and Economics Studies from University of Granada, was graduated in Building Engineering and Master's Degree in Integral Safety in Building from University of Seville and University of Granada. Senior technician in occupational risk prevention. Author of several papers, at Fidesol she currently focuses her research on the technological area.

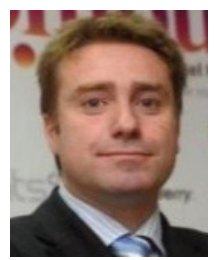

Juan Manuel Lombardo

$\mathrm{PhD}$ in Computer Science from the Pontifical University of Salamanca, was graduated in Economics and Business Administration in the University of Granada, Spain, Diploma of Advanced Studies (DEA) in Economics from UNED, Research Sufficiency in Business Science from the Complutense University of Madrid and Diploma of Advanced Studies (DEA) in Sociology from the Pontifical University of Salamanca. He is CEO at Fidesol and Professor at Andalusia Business School. Dr. Lombardo is the author of numerous articles and research papers published in journals and books of national and international conferences. Visiting Professor at the Private Technical University of Loja (UTPL Ecuador), The National University of the Northeast (Argentina), University Francisco José de Caldas (Colombia), Catholic University of Colombia, Catholic University of Ibarra (Ecuador), University of Lisbon (Portugal) and National Engineering University (Peru). Member of the Knowledge Management committee of AEC (Spanish Association for Quality) and the Institute CICTES (Ibero-American Centre on Science, Technology and Society).

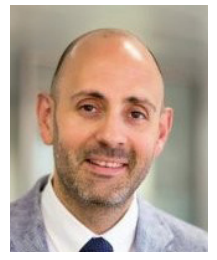

Rubén Gonzalez Crespo

Dr Rubén González Crespo is a full professor in Computer Science and Artificial Intelligence. Currently he is ViceRector of Academic Affairs and Teaching from UNIR. He is $\mathrm{EiC}$ of the International Journal of Interactive Multimedia and Artificial Intelligence (SCIE), and associate editor in several indexed journals. His main research areas are Artificial Intelligence, Accessibility and TEL. He is advisory board member for the Ministry of Education in Colombia and Spain. 